\title{
Non-stationary volume-adsorption model for pumping of vacuum chambers
}

\author{
Leonid Rozanov ${ }^{1, *}$, Aleksandr Kirillov ${ }^{1}$ \\ ${ }^{1}$ Peter the Great St. Petersburg Polytechnic University, Polytechnicheskaya 29, Saint-Petersburg, \\ 195251 Russian Federation
}

\begin{abstract}
The non-stationary mathematical model of pumping of gas, located in the volume and adsorbed on the inner surface of vacuum chambers is considered. A feature of the model is the use of variation of adsorption heat with respect to the logarithm of the degree of coverage obtained from the Frendlich adsorption isotherm. The relations between pressure, gas release, heat of adsorption, the amount of adsorbed gas and the pumping time are calculated. The existence of a regular pumping mode is shown. Experimental verification of the model confirms the theoretical calculations. The model makes it possible to specify the pumping time of International Thermonuclear Experimental Reactor vacuum chambers and to obtain a significant economic effect when selecting pumping means by reducing the safety factor.
\end{abstract}

\section{Introduction}

Vacuum chambers, in addition to gas in the volume, may contain a large amount of gas adsorbed on its internal surfaces. The adsorbed gas, which is energetically bound to the surface, is pumped out more slowly than the gas in the chamber volume. With a high vacuum, the pumping time of the vacuum chamber is almost completely determined by the pumping time of adsorbed gases [1-20]. For large vacuum installations, for example International Thermonuclear Experimental Reactor (ITER), when choosing pumping means by specifying the pumping time, you can reduce the safety factor and get a significant economic effect.

Adsorbed gases mainly consist of water vapors absorbed on the walls of vacuum chambers during their depressurization [1-12]. Oils and organic compounds with a high vapor pressure at room temperature can also make a significant contribution to the total amount of adsorbed gas.

In the literature, diffusion [1] and adsorption [2] mathematical models are presented which determine the amount of absorbed gas at the internal surfaces of the vacuum systems. This work is devoted to development of the adsorption model, which determines the rate of gas removal from the surface by condensation and evaporation processes. In [2], the possibility of using various adsorption equations in an adsorption model for pumping vacuum systems is considered, and it is concluded that it is advisable to use the Temkin isotherm equation for these purposes.

* Corresponding author: Inrozanov@mail.ru 
A discussion of the adsorption model in the literature, for example, in [6], was made on the basis of [2]. The disadvantage of this approach is the use of a linear relation between the adsorption heat and the degree of coverage, which is not fulfilled for the adsorption of water vapors on the walls of vacuum chambers. Experimental data on the adsorption of water on the surface of stainless steel are well described by the Frendlich isotherm equation [3].

In this model, we used the logarithmic dependence of the heat of adsorption on the degree of coverage $[4,5]$. The model allows one to calculate the relation between pressure, the amount of absorbed gas, gas emission, adsorption heat and the pumping time. An experimental study of the curve of water vapor pumping from the surface of the vacuum chamber showed a satisfactory agreement between the theoretical calculations and the experiment.

\section{Volume-adsorption model for pumping of vacuum chambers}

A mathematical model of the volume-adsorption pumping of vacuum chambers can be composed while considering the processes of evaporation, condensation and pumping. The system of differential equations of mass transfer in the chamber volume and on its internal surfaces is represented as:

$$
\left\{\begin{array}{l}
\frac{d a}{d t}+\frac{a}{\tau_{a}}-K p=0 \\
\frac{d p}{d t}+\frac{p}{V / S}+\frac{d a}{d t} \cdot \frac{F}{V}=0 \\
Q_{a}=E-K_{Q} \cdot \lg \left(\frac{a}{a_{m}}\right) \\
q_{a}=\frac{d a}{d t}, \\
S=K_{u} \cdot S_{m}\left(1-\frac{p_{m}+p_{b} / K_{c}}{K_{u} p}\right)
\end{array}\right.
$$

The first differential equation is

$$
\frac{d a}{d t}+\frac{a}{\tau_{a}\left(Q_{a}\right)}-K p=0
$$

where $a$ is specific amount of the adsorbed gas, $\mathrm{m}^{3} \mathrm{~Pa} / \mathrm{m}^{2} ; t$ is time, $\mathrm{s} ; p$ is pressure, $\mathrm{Pa}$;

$$
K=\frac{s \cdot N_{A}}{2.65 \cdot 10^{20} \sqrt{2 \pi M R T}}=\frac{s \cdot 1.08 \cdot 10^{4}}{\sqrt{M T}}, \quad s \quad \text { is probability of condensation, }
$$
$\tau_{a}\left(Q_{a}\right)=\tau_{0} \exp \left(\frac{Q_{a}}{R T}\right)$

$\tau_{0}=1.6 \cdot 10^{-13} \cdot 298 / T, R=8.31 \cdot 10^{3} \mathrm{~J} / \mathrm{kmol}, N_{A}=6.02 \cdot 10^{26} \mathrm{kmol}^{-1}$, and denotes the speed of mass-flow at the internal surfaces of the vacuum chamber.

Differential equation

$$
\frac{d p}{d t}+\frac{S \cdot p}{V}-\frac{d a}{d t} \cdot \frac{F}{V}=0
$$


where $V, F$ and $S$ are volume, internal surface area and effective pumping speed of the vacuum chamber: pumping of the vacuum chambers with adsorbing walls, denotes the mass transfer processes in the volume of the vacuum chamber.

We will use the linear relation between the adsorption heat and the logarithm of the amount of absorbed gas in the form [5]:

$$
Q_{a}=Q_{1}-K_{Q} \lg (\theta)
$$

where

$$
Q_{1}=R T \ln \left(\frac{d^{\frac{1}{m}}}{K \tau_{0} a_{m}^{\frac{1-m}{m}}}\right) \quad K_{Q}=\frac{R T(1-m)}{0.43 \cdot m}
$$

Here $d$ and $m$ are Frendlich adsorption isotherm equation coefficients

$$
a=d \cdot p^{m}
$$

where $a_{m}$ is the amount of gas in the monolayer at a unit of a real surface, $\mathrm{m}^{3} \mathrm{~Pa} / \mathrm{m}^{2}$.

For water at the unprocessed stainless steel at $298 \mathrm{~K}, a_{\mathrm{m}}=0.116 \mathrm{~m}^{3} \mathrm{~Pa} / \mathrm{m}^{2}, d=0.0185, m=$ 0.34 [3]. At $\tau_{0}=1.6 .10^{-13} \mathrm{~s}, s=0.4$ and $K=59$ from (5) it follows that $\mathrm{Q}_{1}=3.34 .10^{7} \mathrm{~J} / \mathrm{kmole}$ and $K_{Q}=1.12 \cdot 10^{7} \mathrm{~J} / \mathrm{kmol}$.

We approximate the effective pumping rate of the pump in the form:

$$
S=K_{u} \cdot S_{m}\left(1-\frac{p_{m}+p_{B} / K_{c}}{K_{u} p}\right)
$$

where $S_{m}$ is the maximum pumping rate, $p_{m}$ is the limiting pump pressure, $K_{u}$ is the coefficient of the pump usage (the ratio between efficient and real rates of pumping), $K_{c}$ is the pump compression coefficient, $p_{b}$ is the release pressure.

Specific gas emission $q_{a}$ we define in the form:

$$
q_{a}=\frac{d a}{d t}
$$

The features of this model are the use of: a logarithmic relationship (4), which allows one to determine the value of adsorption heat according to the degree of surface coverage at any time during pumping out; monomolecular coating of a real rough surface; approximating function (7) for calculation the speed of pumping the camera.

\section{Calculations}

Model (1) allows us to obtain a numerical solution that determines the following characteristics, with respect to the pumping time: pressure, the amount of adsorbed gas, the rate of adsorption gas emission, the heat of adsorption and the effective rate of pumping of the vacuum chamber.

Examples of curves for pumping the water vapor from the vacuum chamber (Fig.1-4) are calculated at $T=298 \mathrm{~K}, v=V / S, f=F / S, p_{m}=10^{-8} \mathrm{~Pa}, p_{6}=10^{0} \mathrm{~Pa}, K_{c}=10^{11}, K_{u}=1, a_{m}=0.116$ $\mathrm{m}^{3} \mathrm{~Pa} / \mathrm{m}^{2}, E=4.4 \cdot 10^{7} \mathrm{~J} / \mathrm{kmol}, K_{Q}=1.12 \cdot 10^{7} \mathrm{~J} / \mathrm{kmol}$. 


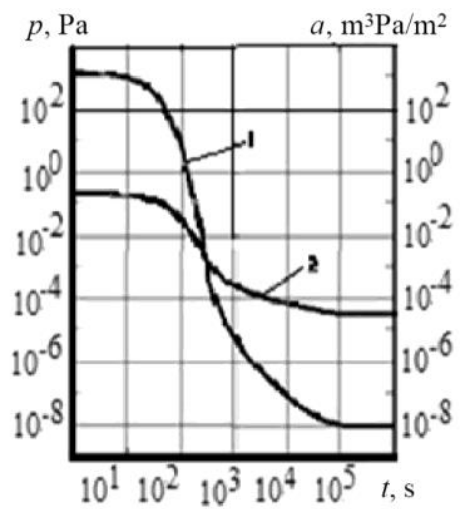

Fig. 1. Curves for water vapor pumping at $v=1 \mathrm{~s}, f=25 \mathrm{~s} / \mathrm{m}, p_{l}=1576 \mathrm{~Pa}: 1-$ pressure, 2 - amount of adsorbed gas.

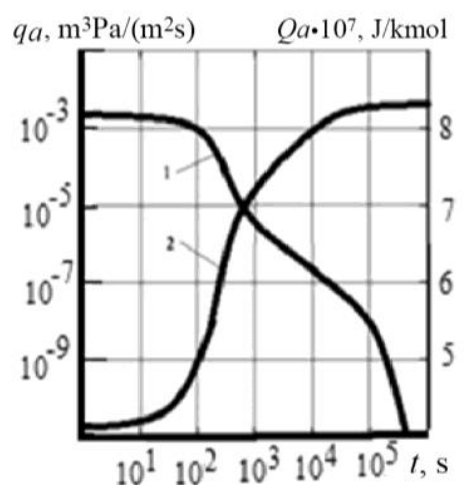

Fig. 2. Curves for water vapor pumping at $v=1 \mathrm{~s}, f=25 \mathrm{~s} / \mathrm{m}, p_{l}=1576 \mathrm{~Pa}: 1-$ specific gas release, 2 adsorption heat.

During pumping (Figure 1), the water vapor pressure decreases almost three orders of magnitude faster than the amount of the adsorbed gas.

The specific adsorption gas emission during pumping non-linearly decreases and tends to zero when the pressure in the chamber tends to the limiting pressure of the pump. The heat of adsorption increases approximately by half from $4.4 \cdot 10^{7}$ to $8 \cdot 10^{7} \mathrm{~J} / \mathrm{kmol}$ during pumping (Figure 2).

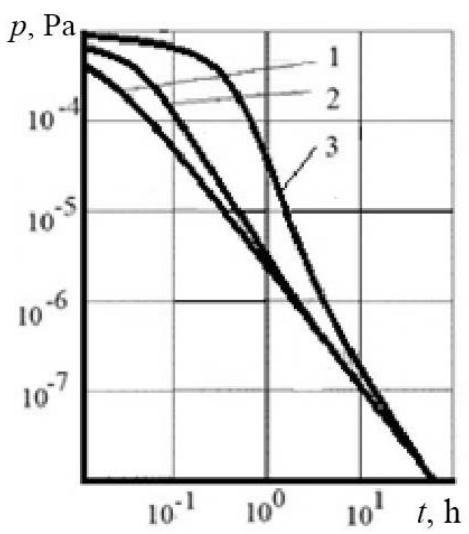

Fig. 3. Curves for water vapor pumping at $f=25 \mathrm{~s} / \mathrm{m}, p_{I}=10^{-3} \mathrm{~Pa}$ for various relative chamber volumes v: $1-1 \mathrm{~s}, 2-100 \mathrm{~s}$, $3-1000 \mathrm{~s}$.

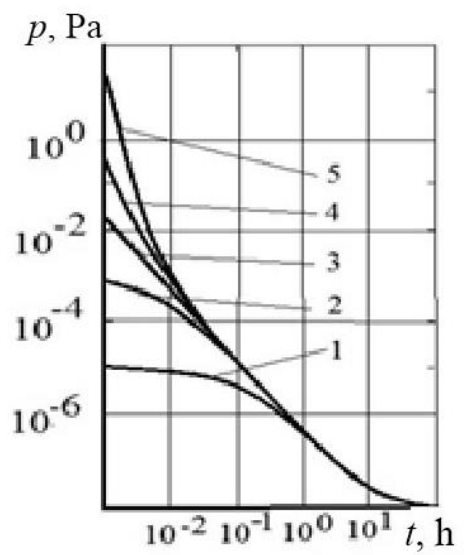

Fig. 4. Curves for water vapor pumping at $f=25 \mathrm{~s} / \mathrm{m}, \mathrm{v}=1 \mathrm{~s}$ for various initial pressures, p1: $1-10^{-5} \mathrm{~Pa}$;

$2-10^{-3} \mathrm{~Pa} ; 3-10^{-2} \mathrm{~Pa}, 4-1 \mathrm{~Pa} ; 5-10 \mathrm{~Pa}$. 
The influence of the chamber volume on the pumping curve (Figure 3) is observed only at the initial stage. For $V / F<100$, the duration of the initial stage does not exceed one hour.

The influence of the initial pressure on the pumping curve is shown in Figure 4. One hour after the start of pumping, the curve practically ceases to depend on the initial pressure.

\section{Steady-state conditions}

When pumping water vapor at $298 \mathrm{~K}$ without noticeable loss of accuracy, the differential equation (2) in model (1) can be replaced with the Frendlich adsorption isotherm equation (6). This means that the adsorption equilibrium on the surface is established faster than the change in pressure in the volume during pumping. Under the regular pumping mode, we will understand the mode in which the pumping process does not depend on the relative volume of the chamber, the limiting pump pressure and the initial pumping pressure.

The mathematical model of the regular pumping mode will take the following form:

$$
\left\{\begin{array}{l}
a(t)=d[p(t)]^{m}, \\
p(t)=\left[\frac{d m F}{3600 S(1-m) t}\right]^{\frac{1}{1-m}}, \\
q_{a}(t)=\frac{p(t) S}{F}, \\
S(t)=K_{u} \cdot S_{m}\left(1-\frac{p_{m}+p_{b} / K_{c}}{K_{u} p(t)}\right), \\
Q(t)=Q_{1}-K_{Q} \lg \left(\frac{a(t)}{a_{m}}\right) .
\end{array}\right.
$$

The simplified model (9) after this replacement has an analytical solution. The pumping time can be obtained from the following equation:

$$
\Delta t=2.3 \cdot v \cdot \lg \frac{p_{1}-p_{m}}{p-p_{m}}+\frac{d \cdot m \cdot f}{1-m}\left[\left(p-p_{m}\right)^{m-1}-\left(p_{1}-p_{m}\right)^{m-1}\right]
$$

where $d$ and $m$ are coefficients of the Frendlich adsorption isotherm equation for water at $298 \mathrm{~K}, p_{m}$ is limiting pressure of the pump, $\mathrm{Pa} ; p_{1}$ is initial pressure, $\mathrm{Pa} ; v=V / S, \mathrm{~s} ; f=$ $F / S, \mathrm{~s} \cdot \mathrm{m}^{-1} ; V$ is the chamber volume, $\mathrm{m}^{3} ; F$ is the area of the internal surface of the chamber, $\mathrm{m}^{2} ; S$ is efficient rate of the chamber pumping expressed in $\mathrm{m}^{3} / \mathrm{s}$ according to the approximation (7). The first term in (10) denotes the pumping time of water vapors, located in the chamber volume, and the second term is for the internal surface.

\section{Experiment}

For experimental verification of the model, an experimental setup was used [4]. The working chamber of the setup had a spherical shape, was made of electropolished stainless steel $12 \mathrm{X} 18 \mathrm{H} 10 \mathrm{~T}$, its volume was 50 liters and the inner surface was $1.2 \mathrm{~m}^{2}$. To 
obtain low and medium vacuum in the setup, the XDS 35i spiral pump was used with a maximum pumping speed of $10 \mathrm{l} / \mathrm{s}$ and a pressure limit of $1 \mathrm{~Pa}$.

The production of high and ultrahigh vacuum was provided by a turbomolecular pump nEXT 300 with a maximum pumping speed of $300 \mathrm{l} / \mathrm{s}$ for nitrogen and a maximum pressure of $10^{-6} \mathrm{~Pa}$ with a spiral pump at the outlet. The cryo-adsorption pump NSJN-01 $\mathrm{M}$ with a pumping rate of $50 \mathrm{l} / \mathrm{s}$ was used as the third stage of pumping. The effective pumping speed of the chamber, taking into account the resistance of pipelines for a spiral pump, was $1 \mathrm{l} / \mathrm{s}$, and for a turbomolecular pump it was $50 \mathrm{l} / \mathrm{s}$.

The total pumping time from atmospheric pressure to $2 \cdot 10^{-6} \mathrm{~Pa}$ is 20 hours and 45 minutes from which are per spiral pump.

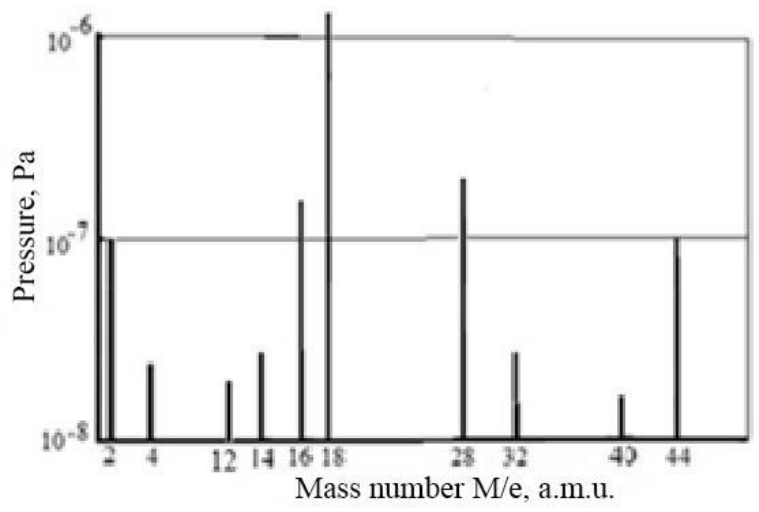

Fig. 5. Mass spectrum of residual gases at the end of pumping.

The main component of the mass spectrum of residual gases (Fig. 5) is water vapor, as one would expect for unheated systems.

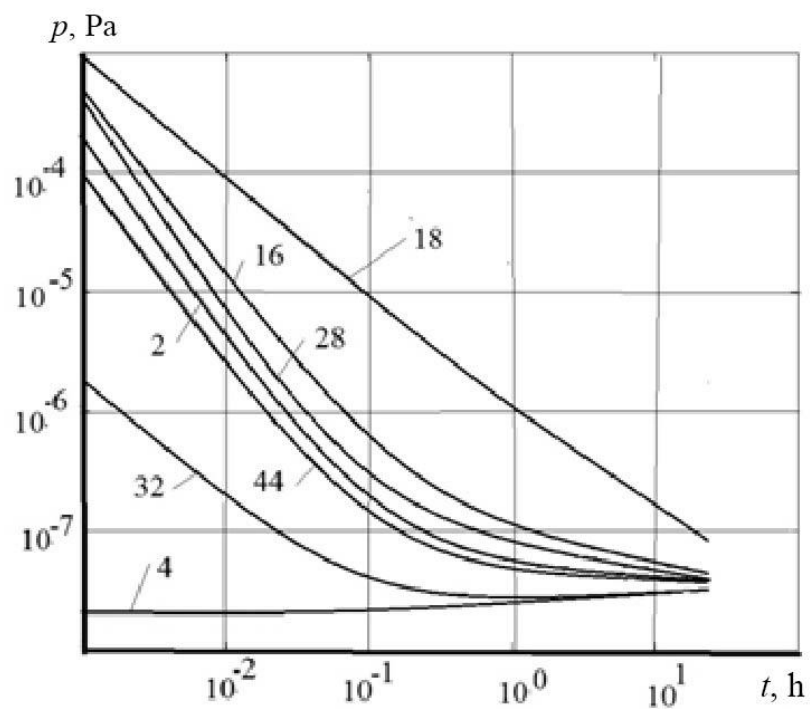

Fig. 6. Pumping curves for separate components of the residual gases.

During the pumping process (Figure 6), in accordance with the adsorption heat, the limiting pressure of water vapor is established more slowly than other components of the 
mixture (more than 20 hours). For other residual gas components, less than 1 hour pumping time is required.

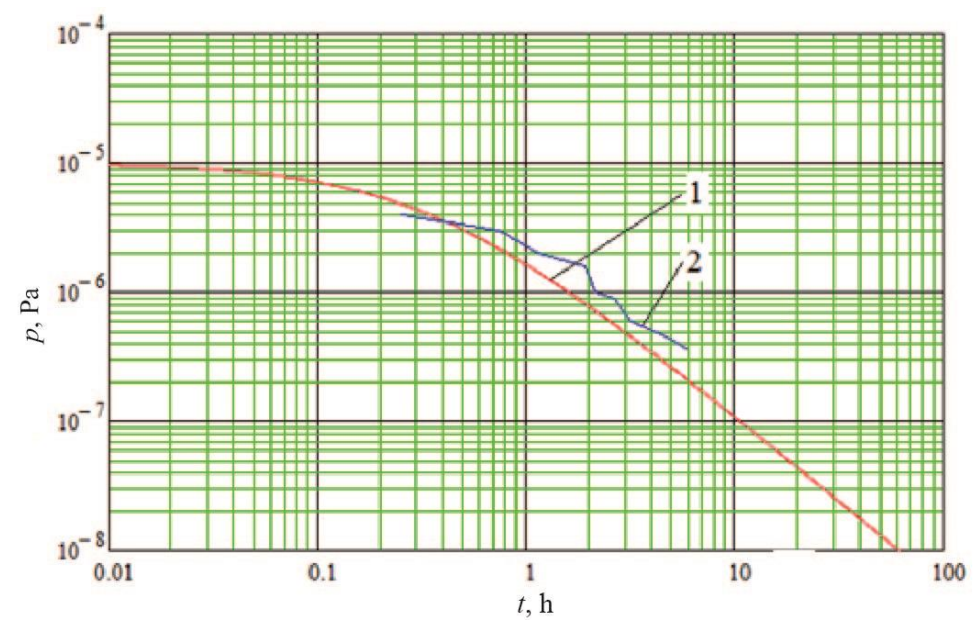

Fig. 7. The curve for water vapor pumping in the experimental setup $(v=V / S=1 \mathrm{~s}, f=F / S=37.5$ $\mathrm{s} / \mathrm{m}$ ) by a turbomolecular pump: 1 - calculated by the model, 2 - experiment.

\section{Conclusions}

We considered the volume-adsorption model for calculation the pumping characteristics of vacuum chambers with absorbing walls. The features of this model are the use of: a logarithmic relation between the adsorption heat and the degree of surface coverage; monomolecular coating of a real rough surface; extended approximation of the effective rate of pumping the chamber from pressure. Using the model for water at $298 \mathrm{~K}$, the relations between pressure, gas evolution, heat of adsorption, the amount of adsorbed gas and the pumping time for a stainless steel vacuum chamber were calculated. The existence of a regular pumping mode is shown, which does not depend on the volume of the chamber and the initial pressure, but is determined only by the area of the inner surface of the chamber. Experimental verification of the model confirms the theoretical calculations of pumping time after the air discharge into the chamber.

\section{Acknowledgements}

The research was performed by a grant of Russian Science Foundation (project No. 1879-10165).

\section{References}

1. M .Li and H.F. Dylla, JVST A12 p.1772. (1994).

2. P.A. Redhead, JVSTA 13p.467 (1995).

3. L.N. Rozanov, Yu.D. Akimov, Journal of applied chemistry of the USSR; 58, 6 13811384 (1985).

4. L.N. Rozanov, IOP Conference Series: Materials and Engineering, 387012067 (2018).

5. L.N. Rozanov, Vacuum, 147 January, 194-198 (2018). 
6. L.N. Rozanov, Vacuum Technique. London and New York, Taylor and Francis (2002).

7. M. Sefa, J. Sentina, B. Erjavec, Vacuum; 98, 3-7 (2013).

8. K. Jousten, Handbook of Vacuum Technology, Wiley-VCH Verlag GmbH, Weinheim, Germany, (2016).

9. H.E. Nuss, Vacuum, 47, N4, 391-395 (1996).

10. V.S. Jilnin, L.N. Jilnina, A.A. Kuzmin, Electronic Engineering, ser. Electric vacuum and gas appliances, 4, 78-80 (1974).

11. B. Garker, Chr. Edellman and M. Ehert, Vacuum, 41, N4, 383-390 (1996).

12. J.R. Chen, J.R. Huang, G.Y. Hsiung, T.Y. Wu and Y.C. Liu, JVST, A 12(4), 17501754 (1994).

13. D. Li, Y. Cheng, Y. Li, L. Zhao, W. Sun, Y. Wang, Vacuum, 136, 14-19 (2017).

14. D. Edwards, J. Vac. Sci. Technol., 14(1), Jan./Feb. (1977).

15. A. Berman, Vacuum, 47(4), 327-332 (1996).

16. B.B. Dayton, 6-th VST, PP, London, 101-109 (1960).

17. J. M. Bur, Foreign liter., 1962.

18. M.D. Malev, Vacuum, 23(2), 43-50 (1973).

19. C. Edelman, Vakuumphysik, Spektrum, Heidelberg Berlin (1998).

20. C. Edelman, I. Kunz, Vacuum, 46(2), 159-163 (1995). 